\title{
Exogenous delivery of organic matter to the early Earth by primitive carbonaceous meteorites and implications for the origin of life \\ ZITA MARTINS
}

IST-ID

Presenting Author: zita.martins@tecnico.ulisboa.pt

The analysis of the organic matter present in primitive carbonaceous asteroids and meteorites provides crucial data on the chemical reactions that occurred on the early solar system. As this carbonaceous material was exogenously delivered to the early Earth, it informs on the chemical evolution of the prebiotic matter that may have contributed to the origin of life in our planet. Primitive carbonaceous meteorites contain $3-5 \mathrm{wt} \%$ of organic carbon, present as insoluble organic matter (IOM) [1], and as soluble organic compounds (SOM) [2]. Different carbonaceous meteorites have organic matter with different abundances, distribution, and chemical composition. It reflects the physical and chemical reactions in the interstellar medium, solar nebula, and/or on their parent bodies. Indeed, organic matter from the initial molecular cloud may have survived the protosolar disk phase, been incorporated into asteroids and comets, and then processed by aqueous alteration on the parent body of carbonaceous meteorites [3]. The analysis of the soluble organic inventory present in these primitive meteorites, and comparison with the one present in interstellar icy grains helps to establish the chemical reactions of the early solar system. In this talk we will discuss the organic content analysis of different carbonaceous meteorites, including one of the least aqueously altered CM chondrite (the Paris meteorite) [4]. We will compare those analysis to the ones of laboratory organic residues, which are analogues of the organic material formed in interstellar icy grains [5]. The laboratory analysis of primitive meteorites, together with present and future space missions to primitive celestial bodies [6] are crucial to understand the origin and evolution of organic matter in the solar system that contributed to the chemistry at the abiotic/biotic transition of the origin of life.

[1] Alexander et al. (2013), Geochimica et Cosmochimica Acta 123, 244.

[2] Martins (2019), In Handbook of Astrobiology, Editor: Kolb. CRC Press. pp. 177.

[3] Messenger (2000), Nature 404, 968.

[4] Martins et al. (2015) Meteoritics \& Planetary Science 50, 926.

[5] Modica et al. (2018), The Astrophysical Journal 865, 41.

[6] Martins et al. (2020), Space Sci Rev 216, 54. 